\title{
Non-thermal laser-induced desorption of metal atoms with bimodal kinetic energy distribution
}

\author{
T. Götz ${ }^{1}$, M. Bergt ${ }^{1}$, W. Hoheisel ${ }^{1}$, F. Träger ${ }^{1}$, M. Stuke ${ }^{2}$ \\ ${ }^{1}$ Fachbereich Physik, Universität Kassel, Heinrich-Plett-Str. 40, D-34132 Kassel, Germany \\ (Fax: + 49-561/804-4518, E-mail: TRAEGER@physik. unikassel.de) \\ ${ }^{2}$ Max-Planck-Institut für biophysikalische Chemie, P.O. Box 2841, D-37018 Göttingen, Germany \\ (Fax: + 49-551/201-1330, E-mail: Mstuke@gwdg.de)
}

Received: 24 May 1996/Accepted: 31 May 1996

\begin{abstract}
Laser-induced desorption of metal atoms at low rate has been studied for pulsed excitation with wavelengths of $\lambda=266,355,532$ and $1064 \mathrm{~nm}$. For this purpose sodium adsorbed on quartz served as a model system. The detached $\mathrm{Na}$ atoms were photo-ionized with the light of a second laser operating at $\lambda=193 \mathrm{~nm}$ and their kinetic energy distribution was determined by timeof-flight measurements. For $\lambda=1064 \mathrm{~nm}$ a distribution typical of thermal bond breaking is observed. If desorption, however, is stimulated with light of $\lambda=266$ or $532 \mathrm{~nm}$, the kinetic energy distribution is non-thermal with a single maximum at $E_{\text {kin }}=0.16 \pm 0.02 \mathrm{eV}$. For $\lambda=355 \mathrm{~nm}$ the non-thermal distribution is even bimodal with maxima appearing at $E_{\text {kin }}=0.16 \pm 0.02$ and $0.33 \pm 0.02 \mathrm{eV}$. These values of the kinetic energies actually remain constant under variation of all experimental parameters. They appear to reflect the electronic and geometric properties of different binding sites from which the atoms are detached and thus constitute fingerprints of the metal surface. The non-thermal desorption mechanism is discussed in the framework of the Menzel-GomerRedhead scenario. The transition from non-thermal to thermal desorption at large fluences of the laser light could also be identified.
\end{abstract}

PACS: 61.80.Ba; 68.55.Jk; 79.20.Ds; 36.40

In recent years, laser-induced surface processes have received growing attention. Understanding of such reactions is not only of scientific interest but opens up the possibility of exploiting them for a large number of applications. An essential process among the variety of lasersurface interactions is non-thermal desorption of atoms, ions and molecules (see e.g. [1]) from the surface of semiconductors $[2,3]$, metals [4-17] or insulators [18]. Even though a large number of experimental and theoretical investigations on non-thermal desorption can be found in the literature, the present understanding of such light-induced reactions is still rather limited. In order to clarify in more detail how atomic or molecular species are liberated from a surface as a result of electronic excitation, experiments along the following lines are of particular value:

i) The incident laser fluence should be kept extremely low so that only subtle changes of the surface morphology are induced. Desorption studies under such conditions of low reaction rate could open the door to correlate the desorption behavior with details of the electronic and geometric structure of different binding sites of the surface. Furthermore, light intensities not sufficient to remove large quantities of material per laser pulse ensure that gas phase collisions of the detached species can be avoided and their genuine kinetic energy distribution is measured. In addition, use of low light fluences minimizes the surface temperature rise and therefore the thermal desorption signal that could obscure observation of non-thermal processes.

ii) It has been concluded from previous experiments that atoms or molecules are preferentially desorbed from "defects" of the surface, i.e. from sites with particularly low coordination numbers $[4,19]$. For this reason the preparation of surfaces with the largest possible number of such binding sites is essential. In other words, the surface under study should have a pronounced but reproducible atomic "roughness" to provide a detectable desorption signal even at low photon fluences.

The present paper reports desorption studies along these lines with the objective to elucidate in more detail which role the surface structure plays in desorption on a microscopic scale. Surfaces with large roughness have been prepared by the deposition of metal atoms on dielectric substrates held at low temperature. For this purpose sodium adsorbed on quartz served as a model system. The deposited atoms form small particles, with the surface defects acting as nucleation centers, a process known as Volmer-Weber growth mode in thin film epitaxy [20]. If the deposition is continued further and further, the clusters finally grow together into a thin film. As will be described in more detail below, laser-induced desorption of atoms from small particles is particularly useful for the investigations reported here, since their surfaces can be 
highly corrugated offering a considerable number of sites with low coordination number from which desorption occurs preferentially. Another essential argument is that the relative number of such "defect" sites can be varied intentionally by changing the size and shape of the clusters. Furthermore, formation of small particles on the surface of a dielectric substrate turns out to ensure reproducible conditions, i.e. the roughness is such that reproducible desorption signals are detected in subsequent experiments. Unlike earlier measurements with continuous-wave laser light [4] the studies described here were also performed out of resonance of surface plasmon excitation with pulsed ultraviolet and infrared radiation. Atoms detached in non-thermal reactions are found and their kinetic energies are determined. Desorption being highly surface specific, it is shown that these kinetic energies essentially reflect the local electronic and geometric properties of different sites on the cluster surface.

\section{Experimental}

The experimental arrangement is shown schematically in Fig. 1. It basically consists of an ultrahigh-vacuum system with the sample, two lasers for stimulating desorption and photo-ionizing the desorption products, and a time-offlight mass spectrometer. A quartz crystal was used as a substrate for small $\mathrm{Na}$ particles. Being mounted to a manipulator, it could be cooled to $80 \mathrm{~K}$ and heated to about $750 \mathrm{~K}$ for cleaning. A thermal atomic beam of $\mathrm{Na}$ atoms with well-defined constant flux was generated and directed onto the substrate in order to deposit a predetermined coverage of atoms onto the surface held at $80 \mathrm{~K}$. The flux of the atomic beam was on the order of $10^{12}$ atoms per second and $\mathrm{cm}^{2}$. It was measured with a quartz crystal microbalance. The deposited atoms assembled into small particles by surface diffusion and nucleation and, at large coverages, formed a continuous, rough metal film. As mentioned above, the experiments reported here were carried out at low coverage, i.e. under conditions where the generated clusters on the surface are well separated and far from growing together. The defects of the substrate surface being "decorated" during the nucleation, the number density of the clusters remained essentially constant during their growth. Consequently, the coverage, i.e. the number of deposited atoms per $\mathrm{cm}^{2}$, and the average particle size are unequivocally related to each other. In the following the mean cluster radius and the metal coverage will therefore often be used synonymously. Under the assumption of a defect density of $10^{10} / \mathrm{cm}^{2}$ the coverage of $5.6 \cdot 10^{14}$ atoms $/ \mathrm{cm}^{2}$ (about one monolayer of $\mathrm{Na}$ ), for example, corresponds to an average cluster radius of $8 \mathrm{~nm}$.

The samples were characterized by optical transmission spectroscopy. For this purpose measurements were carried out with a Xe-arc lamp and a monochromator in the wavelength regime between $\lambda=260$ and $950 \mathrm{~nm}$. Light with s- and p-polarization was used. It is wellknown that the optical spectra of supported clusters are dominated by surface plasmon oscillation with resonances appearing for excitation along the particle axes in the directions parallel and perpendicular to the substrate surface. As described in detail elsewhere [21], measurement

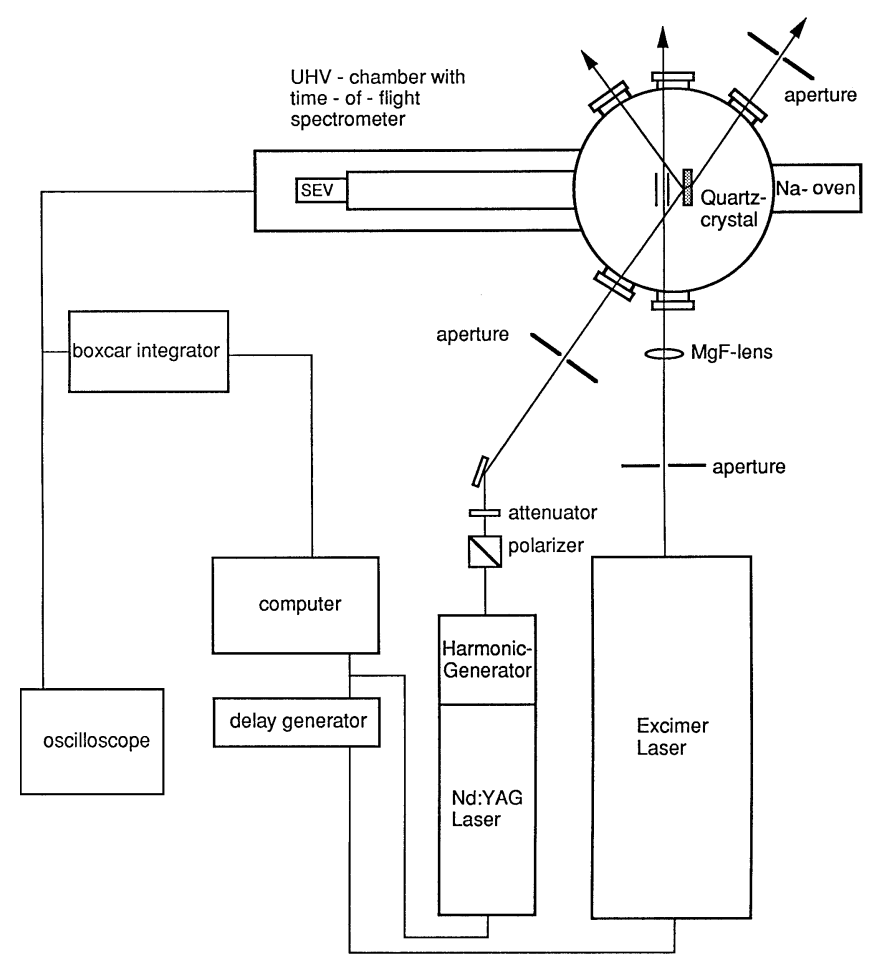

Fig. 1. Experimental arrangement for laser induced desorption studies of metal atoms. Desorption is accomplished by the light of a pulsed (7 ns) Nd:YAG laser and its higher harmonics at $\lambda=1064$, 532,355 and $266 \mathrm{~nm}$. The desorbed atoms are photoionized with the light of an excimer laser fired after a variable delay time and finally identified by a time-of-flight mass spectrometer

of the positions of these two resonances allows one to determine the average size and shape of the metal particles. As a result, the mean radii turned out to range from several up to $50 \mathrm{~nm}$, the axial ratio of the three-dimensional oblate particles decreased with size from 0.5 to 0.2 . As mentioned above, $\mathrm{Na}$ adsorbed on quartz served as model system for the desorption studies described below. Sodium is an ideal candidate for such measurements since the coverage, after completion of an experiment, can be evaporated completely at a temperature of only about $300 \mathrm{~K}$. This made it possible to use the same substrate repeatedly and thus ensured reproducible preparation of the sample as well as reproducible desorption signals. Furthermore, surface plasmon excitation in Na clusters can be stimulated readily with visible light making the above mentioned characterization by means of the optical spectra easily possible.

After preparation and characterization the samples were irradiated with the fundamental or higher harmonics of a Nd:YAG laser at $\lambda=1064,532,355$ and $266 \mathrm{~nm}$ in order to stimulate desorption of atoms from the surface of the $\mathrm{Na}$ particles. The angle of incidence was $50^{\circ}$ with respect to the surface normal and the pulse duration about $7 \mathrm{~ns}$ at a repetition rate of $10 \mathrm{~Hz}$. Laser fluences ranging from 0.1 up to $150 \mathrm{~mJ} / \mathrm{cm}^{2}$ were used. At a distance of $20 \mathrm{~mm}$ in front of the sample the atoms detached from the cluster surface were ionized with the light of an excimer laser operating at $\lambda=193 \mathrm{~nm}$. Its beam was focussed with a magnesiumfluoride lens to a diameter of 
$100 \mu \mathrm{m}$ in the ionization region. The generated ions passed the time-of-flight spectrometer for identification of the mass and were finally detected with a secondary electron multiplier. The time dependent ion signal was processed with a boxcar integrator and stored in a computer. By varying the delay time between the two laser pulses used for desorption and ionization the time-of-flight distributions $I(t)$ of the desorbed $\mathrm{Na}$ atoms could be determined. The kinetic energy distributions $f(E)$ were obtained from the time-of-flight spectra by the relation $f(E)=\mathrm{C} I(t) t^{2}$ where $C$ denotes a constant and $t$ is the variable delay time between the two laser pulses [22]. Data were taken for different fluence, frequency and polarization of the light used for desorption but also in dependence of the metal coverage and for different substrate temperatures.

\section{Results}

\subsection{Desorption with laser light of $\lambda=355 \mathrm{~nm}$}

As an example, Fig. 2 displays a series of three kinetic energy distributions obtained by desorbing atoms with laser light of $\lambda=355 \mathrm{~nm}$. The laser fluence was held constant at $6.6 \mathrm{~mJ} / \mathrm{cm}^{2}$ and the substrate temperature was kept at $T=80 \mathrm{~K}$, the value at which the sample had been prepared. Measurements were performed for increasing Na coverage on the quartz substrate. Surprisingly, at very low integral coverage of $2.2 \cdot 10^{14}$ atoms $/ \mathrm{cm}^{2}$, which corresponds to an average particle size of $R_{\text {ave }}=5 \mathrm{~nm}$, two distinct maxima appear in the kinetic energy distribution. They are located at $E_{\text {kin }}=0.16$ and $0.33 \mathrm{eV}$ (Fig. 2a). The distribution can be approximated by two Gaussians, the sum of which is represented by the solid line. If the coverage is increased to $3.8 \cdot 10^{14}$ atoms $/ \mathrm{cm}^{2}$ (average cluster size of $R_{\text {ave }}=7 \mathrm{~nm}$ ) the integral desorption rate grows. Simultaneously, the amplitudes of the two maxima change (Fig. 2b). Their positions, i.e. the most likely values of the kinetic energies, however, remain constant. The same development continues upon further growth of the coverage to $1.1 \cdot 10^{15}$ atoms $/ \mathrm{cm}^{2}\left(R_{\mathrm{ave}}=10 \mathrm{~nm}\right.$ cluster size $)$. At this point the maximum at $E_{\text {kin }}=0.16 \mathrm{eV}$ is by far predominant in comparison to the second one at $E_{\text {kin }}=0.33 \mathrm{eV}$ (Fig. 2c).

The dependence of the desorption rate on the laser fluence can be seen from Fig. 3. The panels display a series of measurements taken with $\lambda=355 \mathrm{~nm}$ and the same coverage of $3.8 \cdot 10^{14}$ atoms $/ \mathrm{cm}^{2}$ (corresponding to a mean cluster size of $R_{\mathrm{ave}}=7 \mathrm{~nm}$ ). The fluence was increased from $0.3 \mathrm{~mJ} / \mathrm{cm}^{2}$ (Fig. 3a) to values of $6.6 \mathrm{~mJ} / \mathrm{cm}^{2}$ (Fig. 3b), $32 \mathrm{~mJ} / \mathrm{cm}^{2}$ (Fig. 3c) and $52 \mathrm{~mJ} / \mathrm{cm}^{2}$ (Fig. 3d). Whereas only two pronounced maxima appear initially (Fig. $3 \mathrm{a}$ and $\mathrm{b}$ ), the kinetic energy distribution changes drastically, if the fluence grows. At a value of $32 \mathrm{~mJ} / \mathrm{cm}^{2}$ (Fig. 3c), the peak at $E=0.16 \mathrm{eV}$ is not detectable any more. Instead, atoms with very low kinetic energies start to substantially contribute to the signal. Their distribution falls off monotonously and can be fit to an exponential that is represented by the dashed line in Fig. 3c. For an even larger fluence of $52 \mathrm{~mJ} / \mathrm{cm}^{2}$ (Fig. 3d) this exponential decrease by far dominates. Nevertheless, the maximum at $E_{\mathrm{kin}}=0.33 \mathrm{eV}$ can still be recognized.
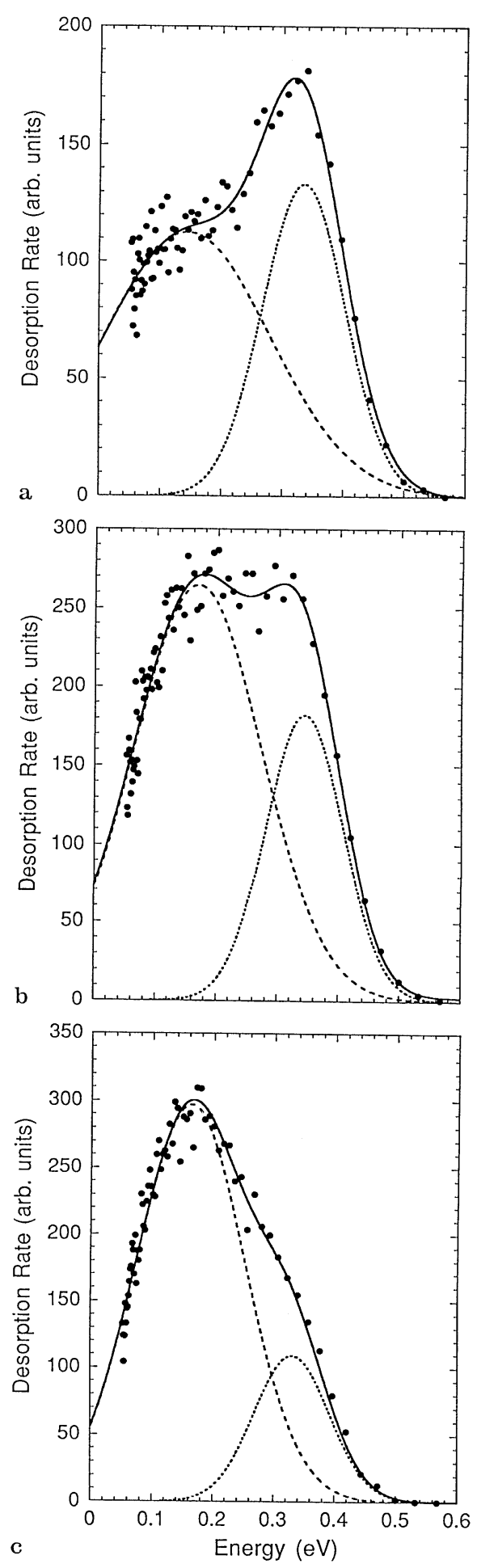

Fig. 2. Kinetic energy distributions of $\mathrm{Na}$ atoms desorbed with pulsed ( $7 \mathrm{~ns}$ ) laser light of $\lambda=355 \mathrm{~nm}$ from the surface of small $\mathrm{Na}$ particles with average radii of a $R_{\text {ave }}=5 \mathrm{~nm}, \mathbf{b} R_{\text {ave }}=7 \mathrm{~nm}$ and c $R_{\text {ave }}=10 \mathrm{~nm}$. The laser fluence was $\Phi=6.6 \mathrm{~mJ} / \mathrm{cm}^{2}$

\subsection{Desorption with laser light of $\lambda=266$ and $532 \mathrm{~nm}$}

In addition to the above measurements at different coverages and laser intensities, the wavelength of the light stimulating desorption has been varied. For this purpose 

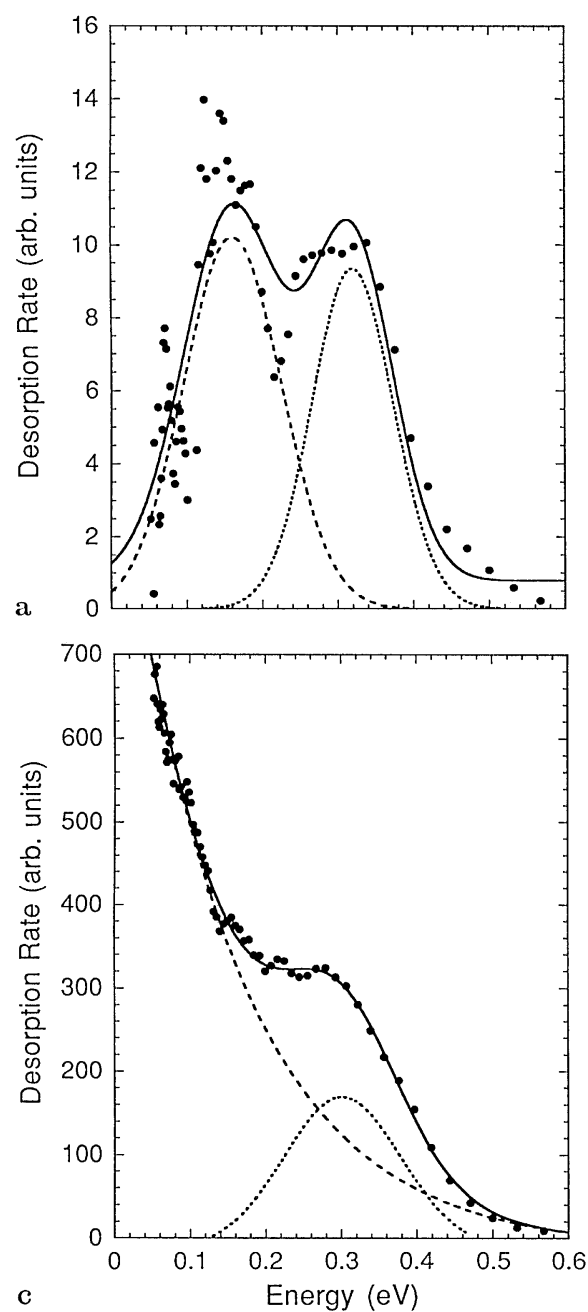
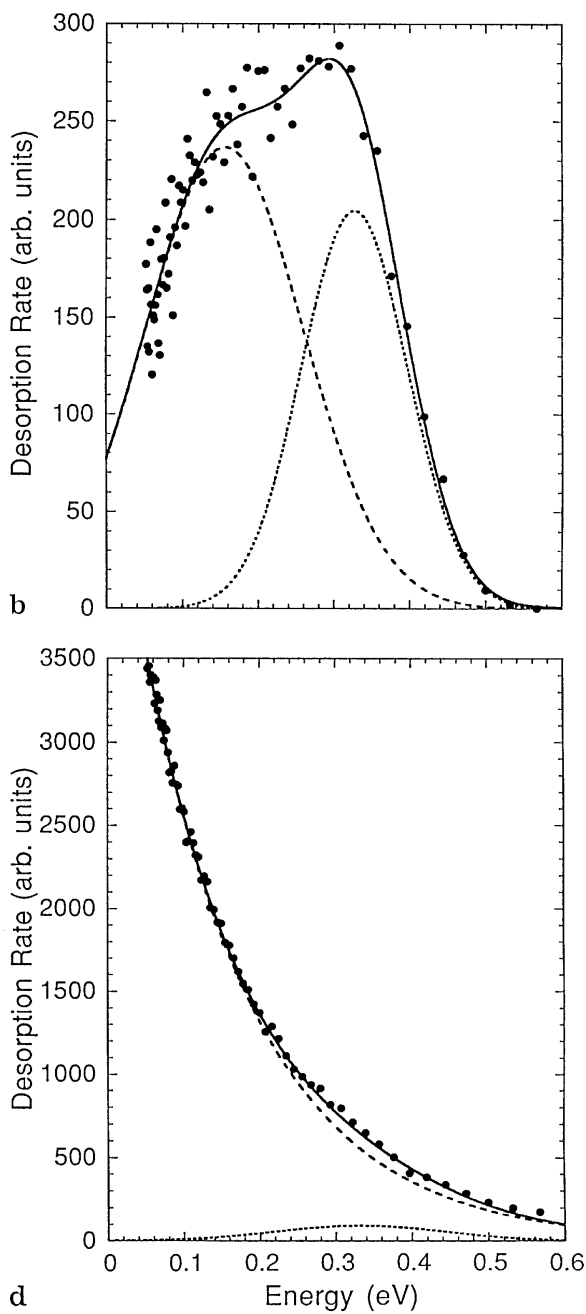

Fig. 3. Kinetic energy distributions of $\mathrm{Na}$ atoms desorbed with pulsed (7 ns) laser light of $\lambda=355 \mathrm{~nm}$ from the surface of small $\mathrm{Na}$ particles with fluences of a $\Phi=0.3 \mathrm{~mJ} / \mathrm{cm}^{2}, \mathbf{b} \Phi=6.6 \mathrm{~mJ} / \mathrm{cm}^{2}$ c $\Phi=32 \mathrm{~mJ} / \mathrm{cm}^{2}$ and $\mathbf{d} \Phi=52 \mathrm{~mJ} / \mathrm{cm}^{2}$. The average particle size was $7 \mathrm{~nm}$ the second $(\lambda=532 \mathrm{~nm})$ and fourth harmonic $(\lambda=266 \mathrm{~nm})$ of the Nd:YAG laser were also used to irradiate the metal particles. The experimental findings are as follows. For both wavelengths, i.e. $\lambda=266$ and $532 \mathrm{~nm}$, only atoms with kinetic energies of around $E_{\text {kin }}=0.16 \mathrm{eV}$ are detected. An example illustrating the results of these measurements is shown in Figure 4. The single maximum found in the distributions is identical to the low-energy signal recorded for $\lambda=355 \mathrm{~nm}$ at the same value of $E_{\mathrm{kin}}$. The bimodal distribution described above is not observed here. As can also be seen from Fig. 4, the position of the maximum is independent of coverage. Furthermore, it does not depend upon the fluence and polarization of the laser light. As for $\lambda=355 \mathrm{~nm}$ the integral desorption rate depends linearly on the fluence of the light stimulating desorption.

\subsection{Desorption with laser light of $\lambda=1064 \mathrm{~nm}$}

If the samples are irradiated with laser light of $\lambda=1064 \mathrm{~nm}$ desorption can be observed only for large fluences above approximately $80 \mathrm{~mJ} / \mathrm{cm}^{2}$ and for large

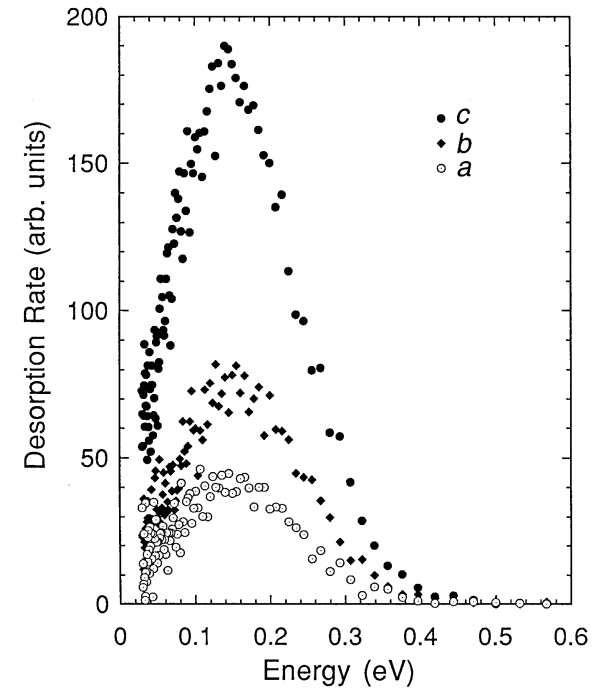

Fig. 4. Kinetic energy distributions of $\mathrm{Na}$ atoms desorbed with pulsed (7 ns) laser light of $\lambda=532 \mathrm{~nm}$ from the surface of small $\mathrm{Na}$ particles for three coverages of a $5.6 \cdot 10^{14}$ atoms $/ \mathrm{cm}^{2}$, b $2.9 \cdot 10^{15}$ atoms $/ \mathrm{cm}^{2}$ and c $5.9 \cdot 10^{15}$ atoms $/ \mathrm{cm}^{2}$. The incident laser fluence was $\Phi=6.6 \mathrm{~mJ} / \mathrm{cm}^{2}$ 
coverages exceeding $9.0 \cdot 10^{15}$ atoms $/ \mathrm{cm}^{2}$. Above the "threshold" value of $80 \mathrm{~mJ} / \mathrm{cm}^{2}$ the desorption rate increases exponentially. Furthermore, the measured kinetic energy distributions of the desorbed atoms follow an exponential similar to the one shown in Fig. 3d. No maxima in the distribution are observed.

\subsection{Desorption after heat treatment of the samples}

Measurements have also been performed after heat treatment of the samples. For this purpose the substrate temperature was raised slowly by using an electrical heater incorporated in the sample holder. Following a predetermined temperature increase, the sample was cooled down to the initial value of $80 \mathrm{~K}$. Subsequently, desorption measurements were carried out. The results of these studies can be summarized as follows. If the temperature rises by about $30 \mathrm{~K}$, the peak at $E_{\text {kin }}=0.16 \mathrm{eV}$ disappears; if the increase is chosen to be larger than $50 \mathrm{~K}$ the maximum at $E_{\mathrm{kin}}=0.33 \mathrm{eV}$ also vanishes. Obviously, the two maxima exhibit different "annealing" behavior. As can be seen from Fig. $3 c$ and d, annealing of the signals also takes place during the light pulse if the sample temperature rises under laser irradiation with large intensities.

\section{Discussion}

We first conclude from the experimental data, that desorption for the three wavelengths of $\lambda=266,355$ and $532 \mathrm{~nm}$ and low fluences obviously occurs as a non-thermal reaction. This is supported by the shape of the kinetic energy distributions of the detected atoms, the absolute energies of 0.16 and $0.33 \mathrm{eV}$ as well as by their independence on the laser fluence. Furthermore, the Na particles only experience a moderate temperature rise under the conditions quoted above [4]. Also, the transition from non-thermal to thermal desorption as a function of laser fluence can be followed and is reflected in the appearance of exponential kinetic energy distributions that gradually replace the curves exhibiting one or even two maxima.

For laser light with $\lambda=1064 \mathrm{~nm}$, on the other hand, the measured kinetic energy distributions are always characteristic of thermal bond breaking and desorption is only observed if the fluence is about two orders of magnitude larger than for the other wavelengths.

Secondly, we notice that the measured kinetic energy values of $E_{\mathrm{kin}}=0.16$ and $0.33 \mathrm{eV}$ are independent of coverage, i.e. particle size, and, as mentioned above, of the light intensity. The two energies actually remain constant under variation of all experimental parameters. Only the desorption efficiency of the two channels changes if, for example, the total coverage of metal atoms on the substrate and therefore the cluster size varies. Furthermore, desorption with the two energies is characterized by different wavelength dependencies. $E_{\text {kin }}=0.33 \mathrm{eV}$ is observed only for $\lambda=355 \mathrm{~nm}, E_{\text {kin }}=0.16 \mathrm{eV}$ also for $\lambda=266$ and $532 \mathrm{~nm}$.

The energy balance of the process is as follows. If desorption is stimulated with light of $\lambda=266,355$ or $532 \mathrm{~nm}$, photon energies of 4.64, 3.48 and $2.32 \mathrm{eV}$,

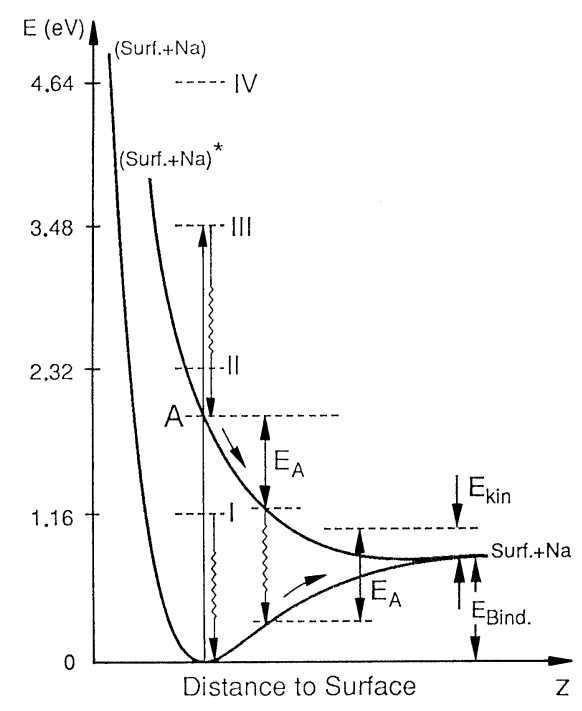

Fig. 5. Schematic of the potential energy diagram of a surface atom illustrating non-thermal desorption of metal atoms within the framework of the Menzel-Gomer-Redhead mechanism. Laser light with $\lambda=266,355$ and $532 \mathrm{~nm}$ initially populates different electronic energy levels denoted by IV $(4.64 \mathrm{eV})$, III $(3.48 \mathrm{eV})$ and II $(2.32 \mathrm{eV})$, respectively. For the sake of clarity only the excitation into state III is shown. Rapid relaxation subsequently leads to a repulsive state A, the atom is accelerated and desorbs with $E_{\text {kin }}=0.16 \mathrm{eV}$. For nearinfrared light with $\lambda=1064 \mathrm{~nm}$ (level $1,1.16 \mathrm{eV}$ ) the antibonding state cannot be reached preventing non-thermal desorption

respectively, are absorbed. These energies, however, are only partially converted into the kinetic energies of the liberated atoms of several tenths of an $\mathrm{eV}$ or needed to supply the binding energy of a surface atoms of about $0.7 \mathrm{eV}$ [23]. Obviously, the initial excitation decays by release of heat into a lower-lying level before desorption takes place. Depending on the photon energy used to stimulate desorption, this process populates a state that is located more than $1 \mathrm{eV}$ above the ground level.

The experimental results allow us to suggest a desorption mechanism along the lines of the Menzel-GomerRedhead scenario [24,25]. This is illustrated in Fig. 5 for the example of atoms detached with $E_{\text {kin }}=0.16 \mathrm{eV}$ by irradiation with $\lambda=266,355$ and $532 \mathrm{~nm}$. Laser light of these wavelengths is absorbed and initially populates different electronic levels denoted as IV, III and II, respectively. For the sake of clarity, only the excitation into state III is shown in Fig. 5. As indicated by the energy balance, each of these excitations is followed by rapid relaxation into a lower-lying state. Since the atoms come off with the same kinetic energy irrespective of wavelength used to stimulate desorption, this intermediate state must be antibonding and identical for all three cases. The atoms are accelerated away from the surface and gain kinetic energy $E_{\mathrm{A}}$. Its value depends upon the lifetime of the repulsive state which in turn determines the kinetic energy of $E_{\text {kin }}=0.16 \mathrm{eV}$ that is finally released. For $\lambda=1064 \mathrm{~nm}$ (corresponding to level 1 with photon energy $1.16 \mathrm{eV}$ ), on the other hand, the antibonding state cannot be reached. As a result, the absorbed photon energy can only be converted into heat causing thermal desorption. 
As mentioned already, light with $\lambda=355 \mathrm{~nm}$ also stimulates desorption of atoms with $E_{\text {kin }}=0.33 \mathrm{eV}$ in addition to those with $E_{\mathrm{kin}}=0.16 \mathrm{eV}$. It therefore appears that a second scenario also gives rise to desorption. It can be regarded as quite similar to the one of the Fig. 5. The involved energy levels, however, are different and excitation with $\lambda$ around $355 \mathrm{~nm}$, presumably resonant, proceeds as the first step.

As mentioned above, the two released kinetic energies of $E_{\text {kin }}=0.16$ and $0.33 \mathrm{eV}$ remain constant under variation of all experimental parameters. Both values obviously constitute fingerprints of the metal surface. The different annealing behavior indicates that desorption of atoms takes place from well defined and different binding sites of the surface. They predominantly exist at low temperature. The variable relative amplitude of the maxima at $E_{\text {kin }}=0.16$ and $0.33 \mathrm{eV}$ reflects a change of their concentrations as the metal coverage on the dielectric substrate is increased. Since both non-thermal signals disappear upon heating, these sites must have particularly low coordination numbers. The electronic wavefunction not being totally delocalized at these "defects", the electron-phononcoupling is reduced in strength, even though prevailing, making possible desorption with finite probability along the lines outlined above. At this point, we would like to emphasize that the potential energy curves drawn in Fig. 5 should only be regarded as plausible assumptions since the local electronic properties at defect sites of the metal surface are not known. Unfortunately, the electronic and geometric signatures of special surface sites embedded in the kinetic energies of the desorbed atoms cannot be readily observed with standard surface science tools like photoemission since contributions originating from the bulk as well as from surface sites of large concentration usually by far dominate the detected signal. Also, these special sites from which desorption is possible cannot be identified by temperature programmed desorption (TPD): before the temperature is reached where evaporation of the particles commences, the sites responsible for non-thermal desorption have disappeared because of annealing. Also, the modern scanning probe microscopies cannot (yet) be utilized to characterize surfaces with such binding sites in detail. In principle, the exact position of an atom on a desorption site with respect to its nearest neighbors would have to be determined together with the local electronic properties.

Even though the sites responsible for desorption cannot be identified unambiguously at present, measurements with different polarization of the laser light provide some hints from where the atoms could be detached. For example, $p$-polarized radiation with $\lambda=532 \mathrm{~nm}$ stimulates desorption more efficiently by $30 \%$ than $s$-polarized light [26]. This indicates that $p$-polarized laser radiation predominantly interacts with a dipole in the direction normal to the surface of the oblate clusters. We therefore suggests that the maximum at $E_{\mathrm{kin}}=0.16 \mathrm{eV}$ can be attributed to adatoms detached from terraces of the particle surface (see also [27, 28]).
Future work will focus predominantly on measuring the wavelength dependence of the two desorption channels with high spectral resolution by using continuously tunable laser light. In addition, extension of the experiments to other metals like $\mathrm{Au}$ and $\mathrm{Ag}$ is in progress. Highly desirable is also to develop methods that allow one to tailormake "non-ideal" surfaces with large numbers of special sites, take images with atomic resolution and characterize the local electronic properties. Finally, the effect presented here could be valuable to remove undesired defects and thus planarize rough surfaces.

Acknowledgements. Financial support of the Fond der Chemischen Industrie (F.T.) and the BMBF under grant number 13N61597 (M. S.) is gratefully acknowledged.

\section{References}

1. Proc. 6th International Workshop Desorption Induced by Electronic Transitions, DIET VI, Nucl. Instrum. Meth. Phys. Res. B 101 (1995)

2. H.T. Liu, Z. Wu: Phys. Rev. Lett. 72, 3891 (1994)

3. P.D. Brewer, M. Späth, M. Stuke: Mat. Res. Soc. Symp. Proc. 334, 245 (1994)

4. W. Hoheisel, M. Vollmer, F. Träger: Phys. Rev. B 48, 17463 (1993)

5. W. Hoheisel, K. Jungmann, M. Vollmer, R. Weidenauer, F. Träger: Phys. Rev. Lett. 60, 1649 (1988)

6. M.J. Shea, R.N. Compton: Phys. Rev. B 47, 9967 (1993)

7. I. Lee, J.E. Parks II, T.A. Callcott, E.T. Arakawa: Phys. Rev. B 39, 8012 (1989)

8. I. Lee, T.A. Callcott, E.T. Arakawa: Phys. Rev. B 47, 6661 (1993)

9. A.M. Bonch-Bruevich, T.A. Vartanyan, Yu.N. Maksimov, S.G. Przhibel'skii, V.V. Khromov: Sov. Phys. JETP 70, 993 (1990)

10. A.M. Bonch-Bruevich, T.A. Vartanyan, Yu.N. Maksimov, S.G. Przhibel'skii, V.V. Khromov: Surf. Sci. 307-309, 350 (1994)

11. H.S. Kim, H. Helvajian: J. Chem. Phys. 95, 6623 (1991)

12. T. Hertel, M. Wolf, G. Ertl: J. Chem. Phys. 102, 3414 (1995)

13. R.S. Mackay, K.H. Junker, J.M. White: J. Vac. Sci. Technol. A 12, 2293 (1994)

14. M. Asscher, F.M. Zimmermann, L.L. Springsteen, P.L. Houston, W. Ho: J. Chem. Phys. 96, 4808 (1992)

15. J.W. Gadzuk, L.J. Richter, S.A. Buntin, D.S. King, R.R. Cavanagh: Surf. Sci. 235, 317 (1990)

16. D.R. Jennison, A.R. Burns, E.B. Stechel: Rev. Solid State Sci. 4, 699 (1990)

17. N.L. Knotek: AIP Conference Proc. 94, 772 (1982)

18. M.A. Schildbach, A.V. Hamza: Phys. Rev. B 45, 6197 (1992)

19. N. Nishi, H. Shinohara, T. Okuyama: J. Chem. Phys. 80, 3898 (1984); M. Vollmer, F. Träger, Surf. Sci. 187, 445 (1987)

20. J.A. Venables: Surf. Sci. 299/300, 798 (1994)

21. T. Götz, W. Hoheisel, M. Vollmer, F. Träger: Z. Physik D 33, 133 (1995)

22. G. Scoles: Atomic and Molecular Beam Methods, Oxford University Press (1988)

23. M. Vollmer, F. Träger: Z. Physik D 3, 291 (1986)

24. D. Menzel, R. Gomer: J. Chem. Phys. 41, 3311 (1964)

25. P.A. Redhead: Can. J. Phys. 42, 886 (1964)

26. M. Bergt, T. Götz, W. Hoheisel, F. Träger: to be published

27. M.D. Thompson, H.B. Huntington: Surf. Sci. 116, 522 (1982)

28. N.D. Lang: Surf. Sci. 299/300, 284 (1994) 